\author{
ANNALES \\ POLONICI MATHEMATICI \\ XLIV (1984)
}

\title{
Non-negative solutions of some non-linear integral equations
}

\author{
by Wojciech Okrasiński (Wroclaw)
}

Abrract. We consider non-negative solutions $u$ of the non-linear convolution equation

$$
W \circ \boldsymbol{K}=\boldsymbol{K} * u,
$$

where the kernel $K$ is a locally bounded non-negative measurable function vanishing on $(-\infty, 0)$ and $W$ is a convex function $(W(0)=0)$ satisfying certain additional conditions.

We give theorems concerning the existence and uniqueness of locally bounded measurable solutions vanishing on $(-\infty, 0]$ and positive on $(0,+\infty)$.

1. Introduction. In papers [2], [3] and [4] are considered non-negative solutions $u$ of the equation

$$
u^{\alpha}(x)=\int_{0}^{x} K(x-\tau) u(\tau) d \tau \quad(\alpha>1)
$$

where $K$ is a non-negative function satisfying certain additional conditions. The solvability of (1.1) is inspected in the function class $M_{0}$ defined as the set of all locally bounded measurable functions vanishing on the non-positive half-line and positive on the positive half-line. The existence and uniqueness of solutions $u$ in $M_{0}$ is showed in [2], [3] and [4]. The proofs are based on the Banach fixed point theorem.

Here we consider the more general equation

$$
W(u(x))=\int_{0}^{x} K(x-\tau) u(\tau) d \tau,
$$

where $W$ is a convex function satisfying certain additional conditions. The kernel $K$ may be of a more general form than in (1.1). Proofs of the existence and uniqueness of solutions in $M_{0}$ will be given without use of the Banach fixed point theorem.

We impose the following assumptions on the kernel $K$ :

$\left(K_{1}\right) K: R \rightarrow R_{+}$is a locally bounded non-negative measurable function $\left(R_{+}=\{x \in R: x \geqslant 0\}\right)$,

$$
\left(K_{2}\right) K(x)=0 \text { for } x<0 \text {, }
$$


$\left(\mathrm{K}_{3}\right)$ there exist numbers $\varepsilon_{1}>0, C_{1}>0$ and $\alpha_{1}>0$ such that $k(x)$ $\geqslant C_{1} x^{x_{1}}$ for $x \in\left[0, \varepsilon_{1}\right]$.

As regards $W$, we assume:

$\left(\mathrm{W}_{1}\right) W: R_{+} \rightarrow R_{+}$is differentiable on $R_{+}$,

$\left(\mathrm{W}_{2}\right) W(0)=W^{\prime}(0)=0$,

$\left(\mathrm{W}_{3}\right) W^{\prime}$ is a strictly increasing continuous function,

$\left(\mathrm{W}_{4}\right) \lim _{x \rightarrow+x} W^{\prime}(x)=+\infty$,

$\left(W_{5}\right)$ there exist numbers $\varepsilon_{2}>0, C_{2}>0$ and $\alpha_{2}>1$ such that $W(x) \leqslant C_{2} x^{x_{2}}$ for $x \in\left[0, \varepsilon_{2}\right]$.

Under these assumptions we will state our results.

2. Some auxiliary lemmas. We introduce certain auxiliary functions and examine their properties.

LEMMA 2.1. Let

$$
H_{1}(x)= \begin{cases}W(x) x^{-1} & \text { for } x>0 \\ 0 & \text { for } x=0\end{cases}
$$

Then $H_{1}$ is a strictly increasing continuous function from $R_{+}$onto $R_{+}$.

Prool. We have

and

$$
\lim _{x \rightarrow 0+} W(x) x^{-1}=\lim _{x \rightarrow 0+} W^{\prime}(x)=0
$$

$$
\lim _{x \rightarrow+\infty} W(x) x^{-1}=\lim _{x \rightarrow+x} W^{\prime}(x)=+\infty
$$

By $\left(W_{3}\right)$ we get that $W(x) x^{-1}$ is strictly increasing. Hence our lemma is true.

COROLlary 2.1. There exists the strictly increasing function $F: R_{+} \rightarrow R_{+}$ inverse to $H_{1}$. The function $F$ is continuous.

Remark 2.1. By $\left(W_{5}\right)$ we have

$$
F(x) \geqslant\left[x / C_{2}\right]^{1 /\left(x_{2}-1\right)} \quad \text { for } x \in\left[0, C_{2} \varepsilon_{2}^{a_{2}^{2}}\right]
$$

LEMMA 2.2. Let

$$
H_{2}(x)=\int_{0}^{x} W(\tau) \tau^{-1} d \tau
$$

Then $\mathrm{H}_{2}$ is a strictly increasing continuous function from $R_{+}$onto $R_{+}$.

The proof of this lemma results from Lemma 2.1 .

COROLlaRY 2.2. There exists the strictly increasing continuous function $G: R_{+} \rightarrow R_{+}$inverse to $\mathrm{H}_{2}$.

Now we give an a priori estimate of the solution $u$ of (1.2). 
Lemma 2.3. If $u \in M_{0}$ is a solution of (1.2), then

$$
u(x) \leqslant F\left(\int_{0}^{x} K(\tau) d \tau\right)
$$

where $F$ denotes the function defined in Corollary 2.1 .

Proof. Let $x>0$. Since

$$
W(u(s)) \leqslant\left[\sup _{s \in I_{x}} u(s)\right] \int_{0}^{s} K(\tau) d \tau \quad \text { for } s \in I_{x},
$$

where $I_{x}=[0, x]$, then

$$
W\left(\sup _{s \in I_{x}} u(s)\right) \leqslant \sup _{s \in I_{x}} u(s) \int_{0}^{x} K(\tau) d \tau
$$

From the last inequality, by Lemma 2.1 , we get

$$
\sup _{s \in I_{x}} u(s) \leqslant F\left(\int_{0}^{x} K(\tau) d \tau\right)
$$

Hence (2.3) is true.

Remark 2.2. Let

$$
\varphi(x)= \begin{cases}0 & \text { for } x<0, \\ F\left(\int_{0}^{x} K(\tau) d \tau\right) & \text { for } x \geqslant 0 .\end{cases}
$$

Then $\varphi$ is a strictly increasing continuous function belonging to $M_{0}$.

Remark 2.3. By $\left(K_{3}\right)$ and Remark 2.1

$$
\varphi(x) \geqslant\left[\frac{C_{1}}{C_{2}}\right]^{1 /\left(\alpha_{2}-1\right)}\left[\frac{1}{\alpha_{1}+1}\right]^{1 /\left(\alpha_{2}-1\right)} x^{\left(\alpha_{1}+1\right) /\left(\alpha_{2}-1\right)}
$$

for $x \in J$, where

$$
J=\left[0,\left(\frac{C_{2}}{C_{1}}\left(\alpha_{1}+1\right) \varepsilon_{2}^{a_{2}}\right)^{1 /\left(\alpha_{1}+1\right)}\right] .
$$

3. Existence of solutions. We define the following operator:

$$
T(f)=W^{-1}(K * f) \quad \text { for } f \in M_{0},
$$

where $W^{-1}$ is inverse to $W$.

Remark 3.1. For $f_{1} \leqslant f_{2}$ we have $T\left(f_{1}\right) \leqslant T\left(f_{2}\right)$.

Remark 3.2. If $f \in M_{0}$ is a non-decreasing function, then $T(f)$ is a non-decreasing function belonging to $M_{0}$.

We will need certain auxiliary theorems in the proof of the existence of non-negative solutions. 
THEOREM 3.1. The function $\varphi$ defined by (2.4) satisfies

$$
T(\varphi) \leqslant \varphi \text {. }
$$

Proof. For $x>0$ inequality (3.2) is equivalent to the following one

$$
(K * \varphi)(x) \leqslant W(\varphi(x)) \text {. }
$$

We shall prove inequality (3.3). By Remark 2.2 we have

$$
(K * \varphi)(x) \leqslant \int_{0}^{x} K(\tau) d \tau F\left(\int_{0}^{x} K(\tau) d \tau\right)
$$

From (3.4) we get

$$
(K * \varphi)(x) \leqslant H_{1}\left(F\left(\int_{0}^{x} K(\tau) d \tau\right)\right) F\left(\int_{0}^{x} K(\tau) d \tau\right) .
$$

By Definition 2.1 we obtain

$$
H_{1}\left(F\left(\int_{0}^{x} K(\tau) d \tau\right)\right) F\left(\int_{0}^{x} K(\tau) d \tau\right)=W\left(F\left(\int_{0}^{x} K(\tau) d \tau\right)\right) .
$$

From (3.5) and (3.6) we get (3.2).

We first examine equation (1.2) in a particular form. We denote by $x_{+}^{a}$ the following function:

$$
x_{+}^{a}=\left\{\begin{array}{ll}
x^{2} & \text { for } x \geqslant 0 \\
0 & \text { for } x<0
\end{array} \quad(\alpha>0) .\right.
$$

This function is in $M_{0}$.

THEOREM 3.2. The equation

$$
u^{\alpha_{2}}(x)=\left(\frac{C_{1}}{C_{2}} x_{+}^{\alpha_{1}} * u\right)(x)
$$

has a solution in $M_{0}$.

Proof. The following formula is known:

$$
\frac{x_{+}^{\mu_{1}}}{\Gamma\left(\mu_{1}+1\right)} * \frac{x_{+}^{\mu_{2}}}{\Gamma\left(\mu_{2}+1\right)}=\frac{x_{+}^{\mu_{1}+\mu_{2}+1}}{\Gamma\left(\mu_{1}+\mu_{2}+2\right)} \quad\left(\mu_{1}, \mu_{2}>0\right),
$$

where $\Gamma$ is the Euler function (see [1]). Let us note that

$$
u_{0}(x)=\left[\frac{C_{1}}{C_{2}} \frac{\Gamma\left(\alpha_{1}+\alpha_{2}+2\right)}{\Gamma\left(\alpha_{1}+1\right) \Gamma\left(\alpha_{2}+1\right)}\right]^{1 /\left(\alpha_{2}-1\right)} x_{+}^{\left(\alpha_{1}+1\right) /\left(\alpha_{2}-1\right)}
$$

is a solution of (3.7). The theorem is proved.

We denote by $\varphi_{0}$ the function

$$
\varphi_{0}(x)=\left[\frac{C_{1}}{C_{2}\left(\alpha_{1}+1\right)}\right]^{1 /\left(a_{2}-1\right)} x_{+}^{\left(a_{1}+1\right) /\left(\alpha_{2}-1\right)}
$$


Then inequality (2.5) may be written as

$$
\varphi(x) \geqslant \varphi_{0}(x) \quad \text { for } x \in J .
$$

We now prove the following theorem:

THEOREM 3.3. The sequence $\left(f_{n}\right)$ of functions from $M_{0}$ defined by

$$
f_{0}=\varphi_{0}, \quad f_{n+1}=T_{1}\left(f_{n}\right) \quad(n=0,1,2, \ldots),
$$

where

$$
T_{1}(f)=\left(\frac{C_{1}}{C_{2}} x_{+}^{\alpha_{1}} * f\right)^{1 / \alpha_{2}} \quad\left(f \in M_{0}\right)
$$

is convergent to $u_{0}$, where $u_{0}$ is given by formula (3.10).

Proof. Let

$$
A=\frac{\Gamma\left(\alpha_{1}+\alpha_{2}+2\right)}{\Gamma\left(\alpha_{1}+1\right) \Gamma\left(\alpha_{2}+1\right)} .
$$

By (3.9) we may write

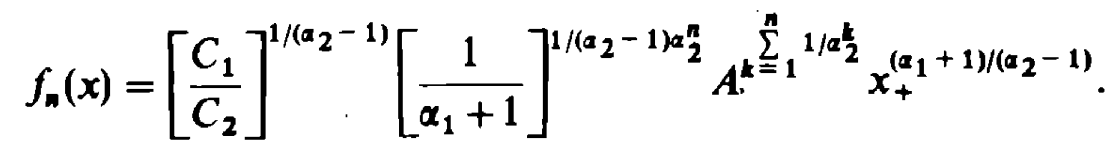

Since $\alpha_{2}>1$, we have

$$
\lim _{n \rightarrow \infty} f_{n}(x)=\left[\frac{C_{1}}{C_{2}} A\right]^{1 /\left(\alpha_{2}-1\right)} x_{+}^{\left(\alpha_{1}+1\right) /\left(\alpha_{2}-1\right)} .
$$

The theorem is proved.

Now we give the theorem about the existence of non-trivial nonnegative solutions of (1.2).

TheOrem 3.4. Equation (1.2) has a solution in the class $M_{0}$.

Proof. We define the sequence $\left(g_{n}\right)$ of functions from $M_{0}$ :

$$
g_{0}=\varphi, \quad g_{n+1}=T\left(g_{n}\right) \quad(n=0,1,2, \ldots) .
$$

Since $\varphi(x) \geqslant \varphi_{0}(x)$ for $x \in J$, then, by $\left(K_{3}\right)$ and $\left(W_{5}\right)$ we have

$$
T\left(g_{n}\right)(x) \geqslant T_{1}\left(f_{n}\right)(x) \quad \text { for } x \in J \text { and } n=0,1,2, \ldots,
$$

where $T_{1}$ is defined by (3.13) and $f_{n}$ by (3.12). By Theorem 3.1 we obtain

$$
T^{n+1}(\varphi) \leqslant T^{n}(\varphi) \quad \text { for } n=0,1,2, \ldots
$$

Then

$$
g_{n+1} \leqslant g_{n} \text { for } n=0,1,2, \ldots
$$

Hence there exists $\lim _{n \rightarrow \infty} g_{n}(x)$. We denote this function by $u(x)$. Since $\left(g_{n}\right)$ is a 
sequence of non-decreasing functions, $u(x)$ is a non-decreasing function. By (3.18) we obtain

$$
u(x) \geqslant u_{0}(x) \quad \text { for } x \in J .
$$

Since $u$ is non-decreasing, then, by (3.21), we see that $u \in M_{0}$. The existence of the solution is proved.

Remark 3.1. Every solution $u \in M_{0}$ of (1.2) is a continuous function. lemma:

4. Uniqueness of non-negative solutions. We prepare the following

LEMMA 4.1. If $M>0$ is a number such that $\varphi(x) \leqslant M$ for $x \in I_{\delta}$, where $I_{\delta}=[0, \delta]$ and $\delta>0$, then

$$
T(M)(x) \leqslant M \quad \text { for } x \in I_{\delta} .
$$

Proof. From our assumptions we obtain

$$
\int_{0}^{x} K(\tau) d \tau \leqslant W(M) M^{-1} \quad \text { for } x \in I_{\delta}
$$

By the definition of $T$ we get

$$
T(M)(x)=W^{-1}\left(M \int_{0}^{x} K(\tau) d \tau\right) .
$$

Since $W^{-1}$ is an increasing function, then, by (4.2) and (4.3), we have

$$
T(M)(x) \leqslant W^{-1}(W(M)) \text {. }
$$

From the last inequality we see that the lemma is true.

Now we state the following theorem:

THEOREM 4.1. If $\varphi(x) \leqslant M$ for $x \in I_{\delta}$, then the sequence $\left(h_{n}\right)$ of functions defined by

$$
h_{0}=M, \quad h_{n+1}=T\left(h_{n}\right),
$$

is convergent to a solution $v$ of (1.2) on $I_{\delta}$ such that $v(x)>0$ for $x \in(0, \delta]$.

Proof. Since, by assumptions,

$$
u(x) \leqslant M \quad \text { on } I_{\delta}
$$

where $u \in M_{0}$ is the solution of (1.2) as in Theorem 3.4, we have

$$
u(x) \leqslant h_{0}(x) \quad \text { on } I_{\delta}
$$

From (4.5) and (4.6) we get

$$
u(x) \leqslant h_{n}(x) \quad \text { for } x \in I_{\delta} \text { and } n=0,1,2, \ldots
$$

By Lemma 4.1 we obtain 


$$
T^{n+1}\left(h_{0}\right)(x) \leqslant T^{n}\left(h_{0}\right)(x) \quad \text { for } x \in I_{\delta} \text { and } n=0,1,2, \ldots
$$

The last inequality is equivalent to the following one:

$$
h_{n+1}(x) \leqslant h_{n}(x) \text {. }
$$

From (4.7) and (4.9) we infer that $\lim _{n \rightarrow x} h_{n}(x)$ exists. We denote this function by $v(x)$. The function $v$ satisfies (1.2) on $I_{\delta}$ and, by $(4.6), v(x)>0$ for $x \in(0, \delta]$.

THEOREM 4.2. Let $m<M$ and $v\left(\delta_{1}\right)=m$, where $\delta_{1} \in(0, \delta)$. Let

$$
k_{0}(x)= \begin{cases}v(x) & \text { for } x \in I_{\delta_{1}}, \\ m & \text { for } x \in\left(\delta_{1}, \delta\right]\end{cases}
$$

Then the sequence $\left(k_{n}\right)$ of functions defined by $k_{n+1}=T\left(k_{n}\right)(n=0,1,2, \ldots)$ is convergent to $v$ on $I_{\delta}$.

Proof. Since $T\left(k_{0}\right)(x)=v(x)$ for $x \in I_{\delta_{1}}$ and $T\left(k_{0}\right)$ is a non-decreasing function, we have

$$
T\left(k_{0}\right)(x) \geqslant T\left(k_{0}\right)\left(\delta_{1}\right)=m \quad \text { for } x \in\left(\delta_{1}, \delta\right] .
$$

Hence

$$
T\left(k_{0}\right)(x) \geqslant k_{0}(x) \quad \text { for } x \in I_{\delta}
$$

From (4.11) we get

$$
k_{n}(x) \leqslant k_{n+1}(x)
$$

But

$$
k_{0}(x) \leqslant M \quad \text { for } x \in I_{\delta}
$$

We have by (4.13)

$$
k_{n}(x) \leqslant h_{n}(x) \leqslant M \quad \text { for } x \in I_{\delta} \text { and } n=0,1,2, \ldots
$$

By (4.12) and (4.14) the sequence $\left(k_{n}\right)$ converges to a function $v$ satisfying equation (1.2) such that

$$
v(x)=v(x) \quad \text { for } x \in I_{\delta_{1}}
$$

and

$$
v(x) \leqslant v(x) \quad \text { for } x \in\left(\delta_{1}, \delta\right] .
$$

Let $x \in\left(\delta_{1}, \delta\right]$. Then by the mean value theorem

$$
\begin{aligned}
v(x) & -v(x) \\
& =T(v)(x)-T(v)(x) \leqslant(K *(v-v))(x)\left[W^{\prime}\left(W^{-1}((K * v)(x))\right)\right]^{-1} .
\end{aligned}
$$

By (4.15) we have 


$$
\begin{aligned}
(K *(v-v))(x)\left[W^{\prime}\left(W^{-1}((K * v)(x))\right)\right]^{-1} & \\
& \leqslant \int_{\delta_{1}}^{x} K(x-\tau)[v(\tau)-v(\tau)] d \tau\left[W^{\prime}\left(v\left(\delta_{1}\right)\right)\right]^{-1}
\end{aligned}
$$

for $x \in\left(\delta_{1}, \delta\right]$. Let $M_{1}=\sup _{x \in I_{\delta}} K(x)$ and

$$
d(v, v)=\sup _{\tau \in\left(\delta_{1}, \delta\right]} e^{-\beta \tau}[v(\tau)-v(\tau)]
$$

where $\beta>0$. We obtain

$$
\int_{\delta_{1}}^{x} K(x-\tau)[v(\tau)-v(\tau)] d \tau \leqslant d(v, v) \int_{\delta_{1}}^{x} K(x-\tau) e^{\beta \tau} d \tau .
$$

From (4.20) we get

$$
\int_{\delta_{1}}^{x} K(x-\tau)[v(\tau)-v(\tau)] d \tau \leqslant d(v, v) \int_{0}^{x} K(x-\tau) e^{\beta \tau} d \tau .
$$

By the commutativity of convolution we have

$$
\int_{0}^{x} K(x-\tau) e^{\beta \tau} d \tau=e^{\beta x} \int_{0}^{x} K(\tau) e^{-\beta \tau} d \tau
$$

From (4.20), (4.21) and (4.22) we get

$$
\int_{\delta_{1}}^{x} K(x-\tau)[v(\tau)-v(\tau)] d \tau \leqslant e^{\beta x} M_{1} \beta^{-1}\left(1-e^{-\beta x}\right) d(v, v) .
$$

From (4.23) we obtain

$$
\int_{\delta_{1}}^{x} K(x-\tau)[v(\tau)-v(\tau)] d \tau \leqslant M_{1} \beta^{-1} e^{\beta x} d(v, v)
$$

for $x \in\left(\delta_{1}, \delta\right]$. Now we assume that

$$
\beta \geqslant 2 M_{1}\left[W^{\prime}\left(v\left(\delta_{1}\right)\right)\right]^{-1} .
$$

From (4.17), by (4.18), (4.23) and (4.25), we obtain

$$
v(x)-v(x) \leqslant \frac{1}{2} e^{\beta x} d(v, v) \quad \text { for } x \in\left(\delta_{1}, \delta\right] .
$$

From the last inequality we infer that

$$
d(v, v)=0 .
$$

Hence $v(x)=v(x)$ for $x \in\left(\delta_{1}, \delta\right]$, too. The theorem is proved. 
Remark 4.1. If a sequence of functions $l_{n}(x)$ is convergent to a solution $l(x)$ of $(1.2)$, then the sequence $l_{n}(x-\eta)$ is convergent to the function $l(x-\eta)$, which is a solution of (1.2).

TheOrem 4.3. Equation (1.2) has the unique solution in $M_{0}$.

Proof. Let $u_{1}, u_{2} \in M_{0}$ be two solutions of (1.2). Let $\delta>0$ be any number and $M=\varphi(\delta)$. Let $\eta>0$ be any number such that $0<\eta<\delta$ and

$$
m=\min \left\{\min _{[\eta, 8]} u_{1}, \min _{[\eta, \delta]} u_{2}\right\}
$$

Let

$$
k_{0}^{\eta}(x)=v(x) \quad \text { for } x \in I_{\delta}
$$

in the case where $v(x)<m$ for $x \in I_{\delta-\eta}$, and let

$$
K_{\delta}^{\prime}(x)= \begin{cases}v(x-\eta) & \text { for } x \in\left[\eta, \delta_{1}\right] \\ m & \text { for } x \in\left(\delta_{1}, \delta\right]\end{cases}
$$

when $v\left(\delta_{1}-\eta\right)=m$. Then

$$
k_{0}^{\eta}(x) \leqslant u_{i}(x) \leqslant M \quad(i=1,2) .
$$

Hence

$$
k_{n}^{\eta}(x) \leqslant u_{i}(x) \leqslant h_{n}(x) \quad(i=1,2),
$$

where $k_{n+1}^{n}=T\left(k_{n}^{\eta}\right)(n=0,1,2, \ldots)$.

Let $n \rightarrow \infty$. From (4.31), by Theorems 4.1, 4.2 and Remark 4.1, we obtain

$$
v(x-\eta) \leqslant u_{i}(x) \leqslant v(x) \quad \text { for } i=1,2 \text { and } x \in I_{\delta} .
$$

Let $\eta \rightarrow 0+$. By the continuity of solutions (see Remark 3.1) we get

$$
v(x) \leqslant u_{i}(x) \leqslant v(x) \quad \text { for } i=1,2 .
$$

The uniqueness is proved.

5. Final remarks. For the proof of the uniqueness assumption $\left(W_{s}\right)$ is not needed. Assumption $\left(\mathbf{K}_{3}\right)$ may be replaced by a more general one, namely:

$\left(K_{3}^{\prime}\right)$ For each $x>0$ the integral

$$
\int_{0}^{x} K(\tau) d \tau
$$

is a positive number.

Under this assumption the uniqueness may be showed. 


\section{References}

[1] Y. Choquet-Bruhat, Distributions. Théorie et problèmes, Masson et $C^{\text {ie }}$, Paris 1973.

[2] J. Goncerzewicz, H. Marcinkowska, W. Okrasiński, K. Tabisz, On the percolation of water from a cylindrical reservoir into the surrounding soil, Appl. Math. 16 (1978), 249-261.

[3] W. Okrasinski, On a non-linear convolution equation occurring in the theory of water percolation, Ann. Polon. Math. 37 (1980), 223-229.

[4] -, On nonnegative solutions of some nonlinear convolution equations, Bull. Acad. Polon. Sci. 26 (1978), 15-18.

Reçu par la Rédaction le 11.12.1980 